\title{
Current status of establishing a venous line in CPA patients by Emergency Life-Saving Technicians in the prehospital setting in Japan and a proposal for intraosseous infusion
}

Kenji Isayama ${ }^{1}$, Toshio Nakatani ${ }^{1 *}$, Masanobu Tsuda $^{1}$ and Akihiko Hirakawa ${ }^{2}$

\begin{abstract}
Introduction: It is important to have a venous line in cardiopulmonary arrest (CPA) patients as an emergency treatment measure in prehospital settings, but establishment of a peripheral venous line is difficult in such patients. This study aimed to investigate the current status of intravenous infusion (IVI) in CPA patients by Emergency LifeSaving Technicians (ELSTs) in Japan. We also considered alternative measures in case IVI was difficult or impossible.

Methods: We investigated a nationwide database between 1 January 2005 and 31 December 2008. From a total of 431,968 CPA cases, we calculated the IVI success rate and related parameters.

The Bone Injection Gun (BIG) and simulator legs (adult, pediatric, and infant) were used by 100 ELSTs selected for the study to measure the time required and the success rate for intraosseous infusion (IOI).

Results: The number of CPA patients, IVI, adrenaline administration, and the IVI success rate in adult CPA patients increased every year. However, the IVI success rate in pediatric CPA patients did not increase. Although adrenaline administration elevated the ROSC rate, there was no improvement in the 1-month survival rate. The time required for IOI with BIG was not different among the leg models. The success rates of IOI with BIG were 93\%, 94\%, and $84 \%$ ( $p<0.05$ vs. adult and pediatric) in adult, pediatric, and infant models, respectively.

Conclusions: The rate of success of IVI in adult CPA patients has been increased yearly in Japan. However, as establishing a peripheral venous line in pediatric patients (1-7 years old) by ELSTs is extremely difficult in prehospital settings, there was no increase in the IVI success rate in such patients. As the study findings indicated $\mathrm{IOI}$ with BIG was easy and rapid, it may be necessary to consider IOI with BIG as an alternative option in case IVI is difficult or impossible in adult and pediatric patients.
\end{abstract}

\section{Introduction}

The Emergency Life-Saving Technicians (ELSTs) system was established in Japan in 1991 as one of the emergency medical service (EMS) systems. ELSTs are permitted to perform endotracheal intubation, intravenous infusion (IVI) of Ringer's lactate solution, and adrenaline administration through a venous line. However, these treatments are allowed only for cardiopulmonary arrest (CPA) patients. Hence, in Japan prehospital care

\footnotetext{
* Correspondence: nakatant@takii.kmu.ac.jp

'Department of Emergency and Critical Care Medicine, Kansai Medical

University, 10-15, Fumizonocho, Moriguchi, Osaka, 570-8507, Japan

Full list of author information is available at the end of the article
}

activities of ELSTs are very limited compared with those in western countries [1-4]. ELSTs are not permitted to perform advanced life support (ALS) such as needle thoracostomy, blood glucose measurements to differentiate hypoglycemic coma, administration of medications other than adrenaline, and intraosseous infusion (IOI) instead of IVI.

Introduction of the Utstein style template enabled the evaluation and comparison of national, regional, and hospital based EMS systems worldwide [5-7]. In January 2005, the Fire and Disaster Management Agency (FDMA) of Japan began accumulating data for out-of- 
hospital cardiac arrest patients using the Utstein template [8].

Among treatments for CPA patients, although the effects of defibrillation, administration of adrenalin, and chest compression have been reported in detail using the Utstein template $[9,10]$, the current status and the effects of IVI by ELSTs have not been reported from Japanese nationwide analysis. Therefore, we focused this study on the analysis of IVI using large-scale data of the Utstein template in Japan.

It is important to have a venous line in CPA patients as an emergency treatment in prehospital settings, but establishment of a peripheral venous line is difficult, especially in dehydrated or hemodynamically unstable patients, particularly so because their peripheral blood vessels are frequently collapsed $[11,12]$. In Japan, among the medical techniques permitted for ELSTs, establishment of a venous line is less frequently attempted and is less successful compared to airway management with devices [13]. Establishing a venous line is essential to administer medications for patients in a prehospital setting. Unfortunately, in many CPA cases that KI encountered as an ELST, it was difficult to establish a peripheral venous line in prehospital settings. Availability of a venous line on arrival at the hospital is helpful for immediate administration of medications and fluids [14]. Obtaining rapid and reliable vascular access is also crucial for the prompt care of critically ill children and adults [15].

However, if it is impossible to perform an immediate IVI in patients, IOI may be an excellent alternative for providing vascular access to administer medications and fluids. Recently, mechanical IOI devices have become more convenient to use compared to manual IOI devices [16,17]. The Bone Injection Gun TM (BIG, WaisMed Ltd., Hertzeliya, Israel) is a small semi-automatic, disposable, spring-loaded IOI device with a trigger. The BIG was the only mechanical IOI device approved in Japan by 2008 . It has been reported that the use of the BIG results in rapid and easy administration of IOI medications and fluids for adults and children with good results $[15,18]$.

The purpose of this study is to investigate the current status of IVI in CPA patients by ELSTs in Japan. Furthermore, we examine the usefulness of IOI with BIG by ELSTs as an alternative option in case IVI is extremely difficult or impossible.

\section{Methods}

\section{Study design (the Utstein style database)}

We investigated a nationwide database for all patients throughout Japan who were transported to hospitals with CPA by ambulances from all Japanese Fire Departments. A total of 431,968 patient records were collected prospectively and accumulated by the FDMA using the Utstein template between 1 January 2005 and 31 December 2008. Some results of this study, such as the rate of return of spontaneous circulation (ROSC) with defibrillation and chest compression, have been reported elsewhere $[8,10]$. We, therefore, focused on the success rate for establishing a venous line by ELSTs. We also compared the rate of ROSC and the 1-month survival rate with or without adrenaline administration by ELSTs in 2008.

In this study, we defined pediatric cases as children between 1-15 years of age. All CPA patients were categorized in age brackets. We also divided pediatric cases into two groups, 1-7 and 8-14 years of age, because adrenaline administration is only indicated for the 8-14year-old group in Japan.

\section{Study design (IOI using $\mathrm{BIG}$ )}

We measured the time required and the success rate for IOI by ELSTs using training BIG and simulator legs (adult, pediatric, and infant). ELSTs tried IOI with BIG in the adult, pediatric, and infant models respectively in spacious surroundings.

\section{Study participants}

In this study, 100 active volunteer ELSTs selected conveniently from 11 fire department headquarters in Japan participated. They had never used BIG previously.

\section{Study instruments}

In this study, we used the adult training BIG (15G, WaisMed Ltd., WMBIG-DEMO-A1, Hertzelia, Israel) and the pediatric training BIG (18G, WaisMed Ltd., WMBIG-DEMO-C2, Hertzelia, Israel). Instruction for usage is the same as for the actual BIG.

\section{$\mathrm{IOI}$ model and penetration site}

Three lower leg models were used in this study. The adult model was made by us. To enable training in BIG needle use, a round hole was cut in the tibial plateau of a mannequin's leg. A small reservoir was fitted into this hole and covered with an artificial bonelike material and silicone rubber "skin." The pediatric model was the lower leg of the Megacode Kid CPR-7500 (no. 23105050, Laerdal Medical AS, Stavanger, Norway). The Megacode Kid is a full-body mannequin reproduction of a 6-year-old boy that is designed for simulation and enables IOI on a pediatric model. The infant model was the lower leg of the ALS baby trainer (no. 08003005, Laerdal Medical AS, Stavanger, Norway). The ALS baby trainer represents a 3-month-old, $5-\mathrm{kg}$ baby and is a simulator designed to provide IOI on an infant model.

The penetration site of the adult leg model was the point two fingerbreadths inside and one fingerbreadth cranial from the tibial tuberosity. The penetration site of pediatric and infant leg models was the point one fingerbreadth inside and one fingerbreadth caudal from the tibial tuberosity. 


\section{Study procedure}

Before commencement of the trial, ELSTs received a brief explanation of a standardized BIG procedure and observed the BIG demonstration by the author. Thereafter, ELSTs practiced once with each model. We measured the time required from selection of the insert point until connecting an infusion line wearing rubber gloves. Successful insertion was defined as a bare needle anchored in a firm upright position in the penetration site.

\section{Questionnaire survey}

ELSTs participating in this study were asked their opinion of whether the device was user friendly, easy to learn, simple, easy to use, and safe to use, and about appropriateness of the BIG for their work environment.

\section{Statistical analysis}

Data were calculated and analyzed using Microsoft Excel 2007 (Microsoft Corp., Redmond, WA). All values were shown with mean \pm standard error of the mean (SEM). Chi-square tests were used to analyze the rate of ROSC, the 1-month rate of survival, and the success rates of each simulator model for IOI with BIG. The time required of each simulator model for IOI with BIG was analyzed according to one-way analysis of variance (ANOVA) followed by Fisher's PLSD (Fisher's Protected Least Significant Difference). The significance level was set at $p<0.05$.

\section{Results}

Analysis of the database

\section{Numbers and rates of IVI success}

Figure 1 shows the numbers of CPA patients, IVI, adrenaline administration, and the rate of successful IVI in the years between 2005 and 2008. The numbers of CPA patients, IVI, adrenaline administration, and the success rate of IVI all increased from 2005 to 2008. Figure 2 compares the numbers of CPA patients, IVI, and the success rate of IVI in all age brackets in 2008. The success rate of IVI in the pediatric age group (1-9 years old) was extremely low at $2.2 \%$. Figure 3 shows the success rate of IVI in CPA patients aged 1-7, 8-14, and above 15 years from 2005 to 2008. Although the success rate of IVI in patients above 15 years of age increased

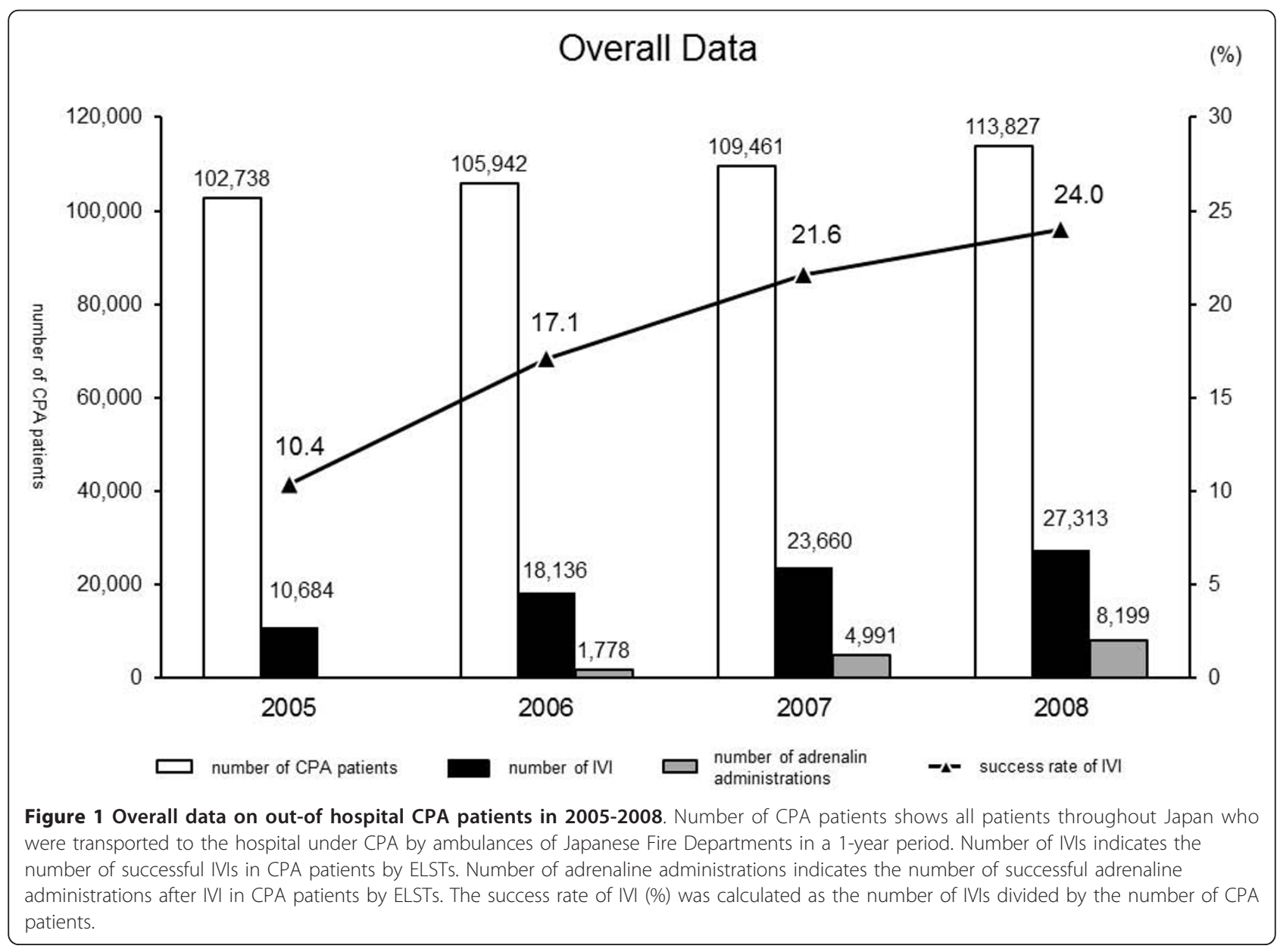




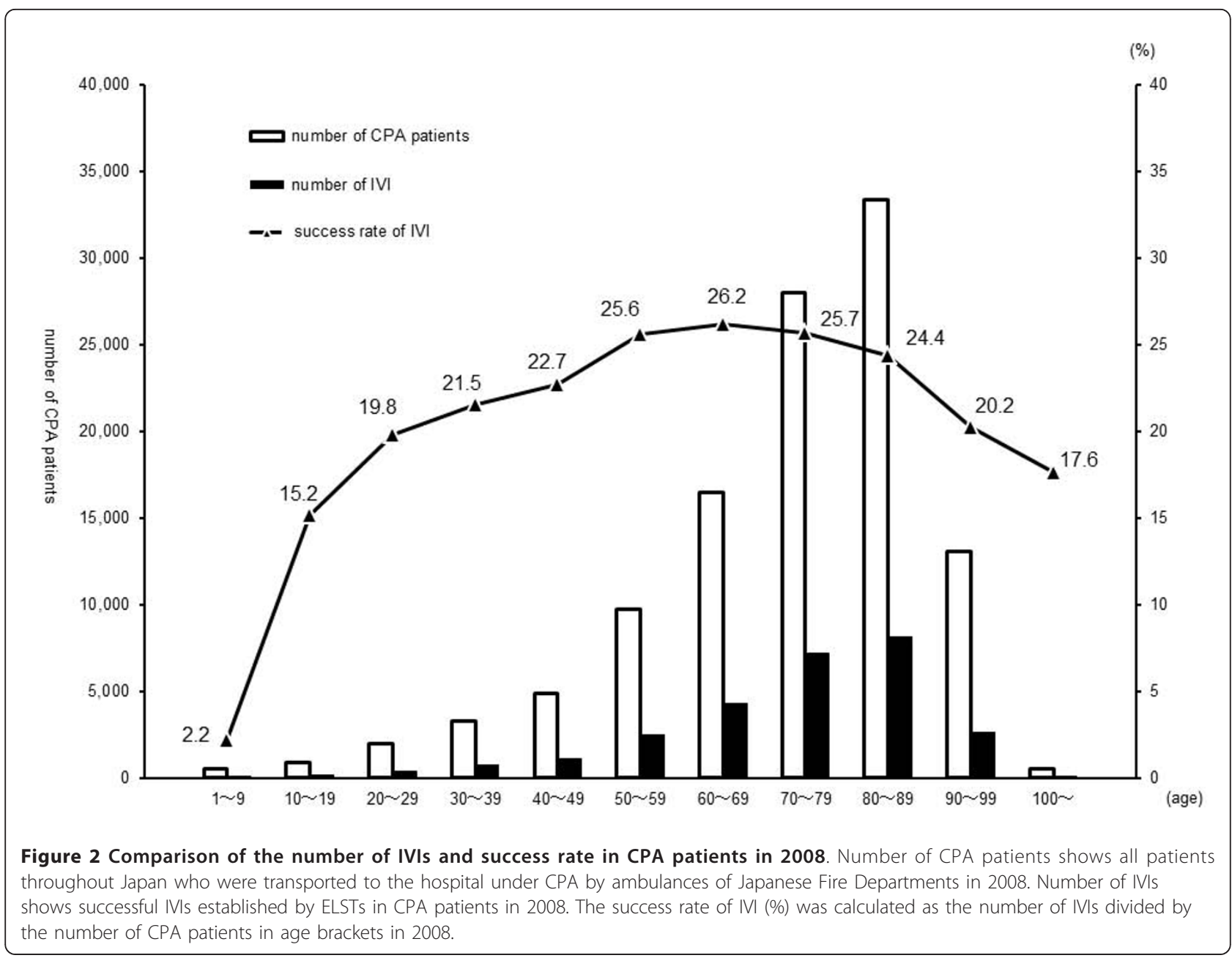

every year, that in pediatric patients (8-14 years old) barely increased from 2005 to 2008 . The success rate of IVI in pediatric patients (1-7 years old) did not increase, and surprisingly slightly decreased in 2008 .

\section{The effect of adrenaline administration}

Table 1 shows the rate of ROSC and 1-month survival rate in CPA patients with or without adrenaline administration by ELSTs in 2008. The rate of ROSC was significantly higher in the group with adrenaline administration compared to the group without adrenaline administration $(p<0.001)$; however, there was no difference in the 1 -month survival rate $(p=0.94)$.

\section{BIG study}

\section{Experimental data}

The BIG study group consisted of 100 participants (volunteer ELSTs) with a mean age of $34.7 \pm 0.64$ years and time in career of $5.2 \pm 0.28$ years.

Table 2 shows the time required and the success rates for IOI with BIG in adult, pediatric, and infant leg models. The time required for performing IOI with BIG among the different leg models was similar. There was a significant difference in the success rate of IOI with BIG in infant leg models when compared to adult $(p<0.04)$ and pediatric $(p<0.03)$ leg models, according to chisquare tests. Sixteen failures occurred in 100 attempts at BIG placement in the infant model.

\section{Questionnaire survey}

The questionnaire survey revealed that ELSTs considered the BIG easy to learn and easy to place. Overall, the BIG was described as satisfactory by $90 \%$ of study participants. All participants expressed great satisfaction with IOI using the BIG, particularly in cases with difficult IVI.

\section{Discussion}

IVI is necessary for fluid infusion and medication administration in acutely affected patients as an emergency treatment in prehospital settings [15]. However, it is not easy to establish a peripheral venous line for various reasons. In the prehospital setting, ELSTs may face additional obstacles, such as expediting patient transport 


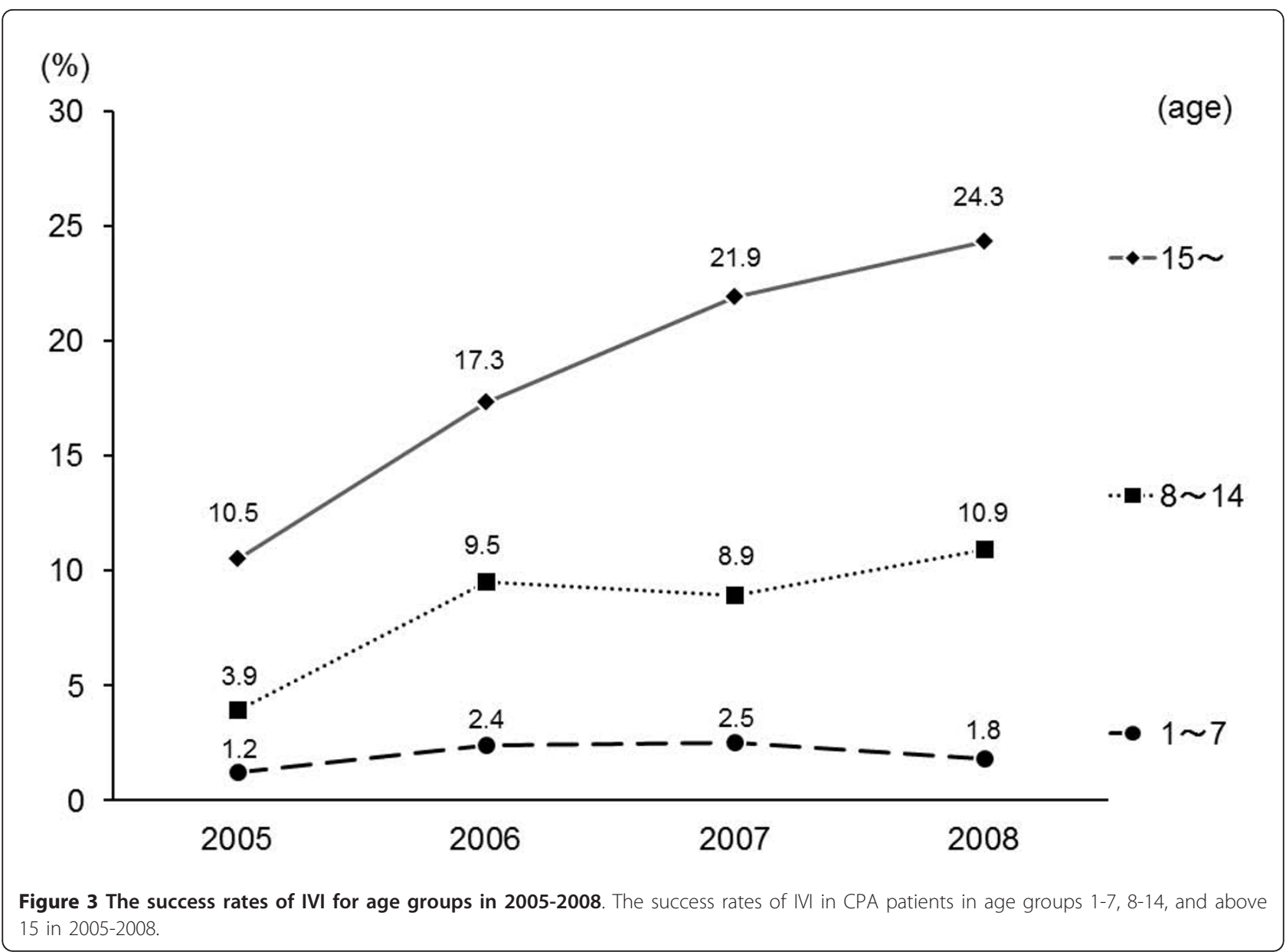

[15]. Usually a hostile environment (inadequate light; a noisy, narrow space; moving ambulance; etc.) makes the introduction of IVI even more difficult. Vascular collapse or inadequate cardiac output may impair access to the peripheral vascular system, and thus hamper emergency medication and fluid administration [19].

Failure rates for IVI in the emergency setting are described as between 10\%-40\% [19]. The average time needed for IVI is reportedly between $2.5-16 \mathrm{~min}$ in patients with difficult IVI [20]. Delays in IVI in the field might be followed by additional delay in the emergency department when reattempting IVI [21]. The resultant time lag for necessary diagnostic and treatment procedures potentially compromises the patient [22]. Prompt transport of CPA patients should not be delayed solely to obtain IVI. IVI should be performed immediately during hospital transport or in a prehospital setting.

The success rate of IVI in CPA patients by ELSTs has increased yearly since authorization of adrenaline administration by ELST in 2006 (Figure 1). However, the extremely low rate of successful IVI in patients aged less than 10 years indicates that IVI is more difficult in pediatric CPA patients than in adults (Figure 2). For example, in 66 pediatric CPA patients, Rosetti et al. demonstrated that experienced emergency department

Table 1 Return of spontaneous circulation (ROSC) and 1-month survival rate after CPA with or without adrenaline administration

\begin{tabular}{ccccc}
\hline & $\begin{array}{c}\text { ROSC } \\
(\mathbf{n = 8 , 1 3 6 )}\end{array}$ & $\begin{array}{c}\text { Non ROSC } \\
(\mathbf{n}=\mathbf{1 0 4 , 3 0 2})\end{array}$ & $\begin{array}{c}\text { 1-month survival rate after CPA } \\
(\mathbf{n}=\mathbf{5 , 9 0 7 )}\end{array}$ & $\begin{array}{c}\text { Non survival } \\
(\mathbf{n}=\mathbf{1 0 6 , 5 3 8 )}\end{array}$ \\
\hline Adrenaline administration & $1,570(18.5 \%)^{* * *}$ & $6,651(81.5 \%)$ & $427(5.2 \%)$ & $7,737(94.8 \%)$ \\
\hline No adrenaline administration & $6,566(6.3 \%)$ & $97,691(93.7 \%)$ & $5,480(5.3 \%)$ & $98,801(94.7 \%)$ \\
\hline
\end{tabular}

ROSC and 1-month survival rate after CPA for adrenaline administration were compared using the chi-square test.

The rate of ROSC was significantly higher in the group with adrenaline administration compared to the group without adrenaline administration; however, there was no difference in the 1 -month survival rate. ${ }^{* * *} p<0.001$ in chi-square test. 
Table 2 Time required and success rates of IOI with BIG

\begin{tabular}{ccc}
\hline & $\begin{array}{c}\text { Time required } \\
\text { Mean } \pm \text { SEM (sec) }\end{array}$ & Success rate \\
\hline Adult & $29.1 \pm 0.63$ & $93 \%(93 / 100)$ \\
\hline Pediatric & $28.7 \pm 0.60$ & $94 \%(94 / 100)$ \\
\hline Infant & $29.3 \pm 0.65$ & $84 \%(84 / 100)^{*}$ \\
\hline
\end{tabular}

The time required for IOI with BIG was compared using one-way analysis of variance (ANOVA) followed by Fisher's PLSD (Fisher's Protected Least

Significant Difference). The success rates of IOI with BIG were compared using the chi-square test.

There was no difference in the time required for performing IOI with BIG among the different leg models. The success rates of IOI with BIG were significantly lower in infant leg models when compared to adult and pediatric leg models.

${ }^{*} p<0.05$ in chi-square test.

personnel required more than 10 min to gain IVI in $24 \%$ of the cases; IVI was never obtained in $6 \%$ of victims [23]. As the success rate of IVI in 1-7-year-old CPA patients did not increase during the study period, this suggests that the rate may not increase in the future (Figure 3). In the expected chaotic early phases of primary resuscitation, timely IVI may be difficult or even impossible in pediatric CPA patients for inexperienced ELSTs. It may be extremely difficult to improve their skills readily for performing IVI in pediatric patients. However, it is necessary to improve IVI rates in pediatric CPA patients.

Current guidelines recommend that IOI should be established in both pediatric and adult emergency patients if it is difficult or impossible to perform an immediate IVI for CPR [24]. The American Heart Association recommends the use of IOI in patients under 6 years of age in need of vascular access who have had two failed IVI attempts or where more than 2 min have elapsed when attempting IVI [25]. Studies have shown that successful IOI within 1-2 min was possible in 72$100 \%$ of patients in the field $[15,26]$. Other studies have demonstrated that IOI can decrease the time needed to perform IVI in pediatric patients under CPA $[25,26]$. IOI of medications achieves adequate plasma concentrations in a time comparable with infusion through central and peripheral intravenous routes for all emergency medications $[27,28]$. However, IOI using a conventional manual IOI needle might be difficult to perform during resuscitation [29].

Mechanical IOI devices have been developed and already have been introduced in many countries; they are an excellent option. The BIG is used in battlefield and prehospital settings to easily and rapidly enable IOI in the USA and Israel [15]. Similarly, in our previous study, IOI with BIG was quick, simple, and unaffected by inexperience or difficult situations for IVI [30]. In addition, in this study, IOI with BIG was easy and rapid (Table 2). In Japan, physicians have recently started to use the BIG in several critical care medical centers and in prehospital settings, such as mobile intensive care units and helicopter emergency medical services.

The results of this study, such as the rate of ROSC with defibrillation and chest compression, have been reported elsewhere $[8,10]$. Compared with patients who received advanced cardiac life support without intravenous medicine administration following cardiac arrest, patients with IVI and medicine administration had a high rate of ROSC but no significant improvement in long-term survival rate [31]. Similarly, in this study, although the adrenaline administration increased the rate of ROSC, there was no difference in the 1-month survival rate (Table 1). However, in Japan the rate of successful IVI in adult CPA patients by ELSTs is low compared to western countries, particularly in pediatric CPA patients where the rate is even lower, and the rate of adrenaline administration is considerably lower (Figure 1). Therefore, we suggest that, first, it is necessary to improve the rate of successful IVI and adrenaline administrations, and subsequently, it should be considered if adrenaline administration is effective or not in CPA patients in prehospital settings in Japan (Figure 1 and Table 1).

IVI in pre-CPA patients is an emergency treatment given by ELSTs. However, for ELSTs, it is difficult to perform IVI in pre-CPA or profound shock patients because of peripheral vein collapse. IOI with BIG may be effective especially in cases where IVI is very difficult or impossible such as in pediatric CPA patients or preCPA patients.

It is reported that the success rate of IOI was $25 \%$ in children aged less than 1 year, 100\% in children aged 12 years, $86 \%$ in children $3-9$ years old, and $74 \%$ in patients above 10 years of age [32]. The BIG may be effective in both adults and children, except for children aged 0-11 months [32]. In this study, the success rate of IOI with BIG in the infant leg model was significantly lower compared to adult and pediatric model legs (Table 2). Because the penetration site in an infant leg is particularly small and narrow, IOI with BIG in infants would be more difficult than in adult and pediatric patients. In fact more failures occurred in BIG placement in the infant leg model. Therefore, close attention should be paid to IOI with BIG in infants.

Our study has certain limitations. We did not compare the BIG to other mechanical devices or manual IOI needles. As the model legs we used in this study are not actual human legs, this experimental data may not similarly reflect the situation for an actual human leg in emergency cases. However, results of our study at least would indicate the definite usefulness of IOI with BIG by ELSTs, particularly in those with difficult IVI conditions. 
23. Rosetti V, Thompson BM, Aprahamian C, Mateer JR, Aprahamian C: Difficulty and delay in intravascular access in pediatric arrests. Ann Emerg Med 1984, 13:406.

24. Biarent D, Bingham R, Eich C, López-Herce J, Maconochie I, RodríguezNúñez A, Rajka T, Zideman D: European Resuscitation Council Guidelines for Resuscitation 2010: Section 6. Paediatric life support. Resuscitation 2010, 15:1364-1388.

25. Kanter RK, Zimmerman JJ, Strauss RH, Stoeckel KA: Pediatric emergency intravenous access: evaluation of a protocol. Am J Dis Child 1986, 140:132-134

26. Calkins MD, Fitzgerald G, Bentley TB, Burris D: Intraosseous infusion devices: A comparison for potential use in special operations. J Trauma 2000, 28:1068-1074.

27. Wenzel V, Lindner KH, Augenstein S, Voelckel W, Strohmenger HU, Prengel AW, Steinbach G: Intraosseous vasopressin improves coronary perfusion pressure rapidly during cardiopulmonary resuscitation in pigs. Crit Care Med 1999, 27:1565-1569.

28. Orlowski JP, Porembka DT, Gallagher JM, Lockrem JD, VanLente F: Comparison study of intraosseous, central intravenous, and peripheral intravenous infusion of emergency drugs. Am J Dis Child 1990, 144:112-117.

29. Brenner T, Bernhard M, Helm M, Doll S, Völkl A, Ganion N, Friedmann C, Sikinger M, Knapp J, Martin E, Gries A: Comparison of two intraosseous infusion systems for adult emergency medical use. Resuscitation 2008, 78:314-319.

30. Isayama K, Hirakawa A, Murao Y, Nakatani T: Studies on the present condition of peripheral intravenous access by emergency medical technicians and on the usefulness of the Bone Injection Gun ${ }^{\mathrm{T}}$. J Jpn Soc Emer Med 2010, 13:690-696, in Japanese.

31. Olasveengen TM, Sunde K, Brunborg C, Thowsen J, Steen PA, Wik L: Intravenous drug administration during out-of-hospital cardiac arrest: a randomized trial. JAMA 2009, 302:2222-2229.

32. Gerritse BM, Scheffer GJ, Draaisma JM: Prehospital intraosseous access with the bone injection gun by a helicopter-transported emergency medical team. J Trauma 2009, 66:1739-1741.

doi:10.1186/1865-1380-5-2

Cite this article as: Isayama et al: Current status of establishing a venous line in CPA patients by Emergency Life-Saving Technicians in the prehospital setting in Japan and a proposal for intraosseous infusion. International Journal of Emergency Medicine 2012 5:2.

\section{Submit your manuscript to a SpringerOpen ${ }^{\mathcal{O}}$ journal and benefit from:}

- Convenient online submission

- Rigorous peer review

- Immediate publication on acceptance

- Open access: articles freely available online

- High visibility within the field

- Retaining the copyright to your article

Submit your next manuscript at $\gg$ springeropen.com 